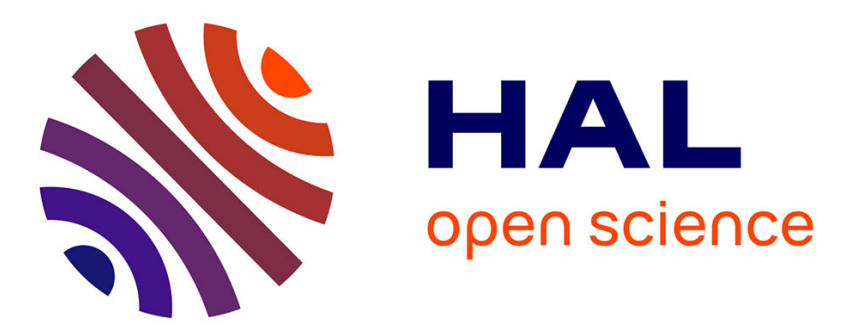

\title{
Normes et valeurs dans le discours des enseignants du primaire : entre pluralité et contradictions internes.
} Jacques Crinon, Catherine Delarue-Breton

\section{To cite this version:}

Jacques Crinon, Catherine Delarue-Breton. Normes et valeurs dans le discours des enseignants du primaire : entre pluralité et contradictions internes.. Recherche et formation, 2018, 10.4000/rechercheformation.4022 . hal-02376024

HAL Id: hal-02376024

https://hal-normandie-univ.archives-ouvertes.fr/hal-02376024

Submitted on 26 Nov 2019

HAL is a multi-disciplinary open access archive for the deposit and dissemination of scientific research documents, whether they are published or not. The documents may come from teaching and research institutions in France or abroad, or from public or private research centers.
L'archive ouverte pluridisciplinaire HAL, est destinée au dépôt et à la diffusion de documents scientifiques de niveau recherche, publiés ou non, émanant des établissements d'enseignement et de recherche français ou étrangers, des laboratoires publics ou privés. 


\title{
NORMES ET VALEURS DANS LE DISCOURS DES ENSEIGNANTS DU PRIMAIRE : ENTRE PLURALITÉ ET CONTRADICTIONS INTERNES
}

\author{
Jacques Crinon et Catherine Delarue-Breton
}

E.N.S. Editions | « Recherche \& formation »

2018/2 n $88 \mid$ pages 17 à 32

ISSN 0988-1824

Article disponible en ligne à l'adresse :

https://www.cairn.inforevue-recherche-et-formation-2018-2-page-17.htm

Distribution électronique Cairn.info pour E.N.S. Editions.

(C) E.N.S. Editions. Tous droits réservés pour tous pays.

La reproduction ou représentation de cet article, notamment par photocopie, n'est autorisée que dans les limites des conditions générales d'utilisation du site ou, le cas échéant, des conditions générales de la licence souscrite par votre établissement. Toute autre reproduction ou représentation, en tout ou partie, sous quelque forme et de quelque manière que ce soit, est interdite sauf accord préalable et écrit de l'éditeur, en dehors des cas prévus par la législation en vigueur en France. Il est précisé que son stockage dans une base de données est également interdit. 


\section{Recherche et formation}

$88 \mid 2018$

Savoirs et normes pour enseigner

\section{Normes et valeurs dans le discours des enseignants du primaire : entre pluralité et contradictions internes}

Norms and values in the discourse of primary school teachers: plurality and internal contradictions

Jacques Crinon et Catherine Delarue-Breton

\section{OpenEdition \\ Journals}

Édition électronique

URL : http://journals.openedition.org/rechercheformation/4022

DOI : 10.4000/rechercheformation.4022

ISSN : 1968-3936

Éditeur

ENS Éditions

Édition imprimée

Date de publication : 30 septembre 2018

Pagination : 17-32

ISBN : 979-10-362-0173-8

ISSN : 0988-1824

Distribution électronique Cairn

\section{CAIRN $\| N F C$}

CHERCHER, REPÉRER, AVANCER

Référence électronique

Jacques Crinon et Catherine Delarue-Breton, « Normes et valeurs dans le discours des enseignants du primaire : entre pluralité et contradictions internes », Recherche et formation [En ligne], 88 | 2018, mis en ligne le 01 janvier 2023, consulté le 22 juillet 2019. URL : http://journals.openedition.org/ rechercheformation/4022 ; DOI : 10.4000/rechercheformation.4022 


\title{
Normes et valeurs dans le discours des enseignants du primaire : entre pluralité et contradictions internes
}

\author{
Jacques Crinon \\ Université Paris-Est-Créteil, Centre interdisciplinaire de recherche, culture, éducation, formation, \\ travail, équipe Éducation et scolarisation (CIRCEFT-Escol, EA 4384)
}

Catherine Delarue-Breton

Université de Rouen-Normandie, laboratoire Dynamique du langage in situ (DYLIS, EA 7474)

RÉSUMÉ : Les normes qui sous-tendent l'action et la pensée des enseignants, compromis entre des normes externes et leurs croyances, sont étudiées ici à partir d'une enquête par questionnaire auprès de 163 enseignants du primaire. Nous analysons les réponses aux questions ouvertes à travers les mots utilisés par les enseignants, en recourant à des statistiques lexicales. Les emplois des mots "élèves" et "temps", très fréquents dans les réponses, font apparaître plusieurs contradictions, qui renvoient à la fois à des dilemmes constitutifs du métier et à un éclatement des repères.

MOTS-CLÉS : enseignant, normes éducatives, discours, école primaire, pratique pédagogique

\section{Des normes pour enseigner}

L'école est un univers normatif, régi par un ensemble de textes qui s'imposent à ses acteurs et tout particulièrement aux enseignants. Les instructions officielles déterminent des objectifs, des contenus d'enseignement, une organisation de ces contenus par rapport auxquels les enseignants doivent rendre des comptes, même si des types de régulation alternatifs, notamment par projets locaux, laissant plus de place à l'initiative des acteurs, viennent aujourd'hui concurrencer avec plus ou moins de succès le modèle vertical et hiérarchique (Buisson-Fenet et Dutercq, 2015).

Dans les faits cependant, l'interprétation de ces normes est sujette à variations : les enseignants jouissent d'une "liberté pédagogique", ils ont le choix des moyens pour appliquer les objectifs fixés. Enfin, au-delà des prescriptions primaires, explicites, l'institution elle-même est porteuse de conceptions du métier et de l'élève qui ne sont pas sans influence sur le travail réel des enseignants. Les pratiques effectives ne reposent donc pas seulement sur les injonctions formelles de l'institution, mais aussi sur des croyances 
(Wanlin et Crahay, 2012), des autoprescriptions (Goigoux, 2007) ou des convictions, fruits de compromis qui ne sont pas purement individuels mais souvent partagés localement ou par des segments de la profession. Il y a une dimension collective et autorégulée de ces "normes et règles qui ne sont pas seulement un reflet, même détourné et aménagé, des normes institutionnelles" (Nonnon et Goigoux, 2007, p. 21). De plus, nous formulons I'hypothèse, pour les individus, d'un "feuilletage normatif " ", lié au fait qu'au-delà des conceptions ou normes affichées qui permettent d'affirmer l'appartenance à un corps, d'autres normes, d'autres croyances ou d'autres conceptions, plus implicites, en lien avec la pratique et ses difficultés inhérentes, mais qui viennent contredire les premières, agissent de manière souterraine.

On sait aujourd'hui, grâce à l'abondance de travaux francophones, mais surtout anglophones sur la question (Crahay, Wanlin, Issaieva et Laduron, 2010), que les conceptions des enseignants, parce qu'elles sont "encapsulées" dans l'expérience, sont peu aisées à distinguer des connaissances qu'ils ont sur le métier, les élèves, les apprentissages, la discipline enseignée etc. (Delarue-Breton, 2016; Delarue-Breton et Dolignier, 2016). Lorsque nous parlons de conceptions ou de représentations des enseignants, il faut également entendre que celles-ci contiennent des éléments de savoir, et sont simultanément propres et partagées par la communauté des enseignants. Elles relèvent d'une épistémologie personnelle qui dans le même temps reflète ce qui est partagé au sein d'une communauté discursive, d'où des tensions, voire des conflits internes. Ceux-ci sont liés à la confrontation entre savoirs théoriques et savoirs issus de l'expérience propre, qui ne convergent pas toujours. Ces conceptions ont d'abord une fonction d'homogénéisation et de stabilisation de ce qui est intrinsèquement hétérogène, instable, mouvant. Mais elles ont aussi une fonction identitaire, qui permet d'attester de l'appartenance à un groupe social (Crahay et al., 2010), qui peut d'ailleurs se traduire aussi bien par l'adhésion que par la distanciation à des normes ou à des valeurs, qui, quoi qu'il en soit, font partie du bagage de l'enseignant. Les normes des enseignants sont ainsi entendues comme principes autoprescrits, et sont analysées comme résultant de compromis entre normes externes et conceptions/croyances.

Le but de cet article est d'explorer ces normes et conceptions, et leur complexité, chez des enseignants de l'école primaire en France à partir d'une enquête par questionnaires leur demandant de déterminer et de justifier une manière de faire, face à une série de situations professionnelles qui leur étaient soumises. Ces justifications font l'objet d'une analyse de discours.

Le parti pris retenu pour cette étude est en effet, à la suite de Clot (2010), que la pratique peut s'analyser bien au-delà - et donc en dehors - de la pratique elle-même, et à partir d'autres procédés méthodologiques que l'observation de celle-ci. Nous postulons

1 Nous proposons cette notion par analogie avec celle de feuilletage énonciatif (voir par exemple Détrie, Siblot et Verine, 2001), qui rend compte de l’hétérogénéité du sujet parlant. 
que l'analyse des discours sur la pratique est susceptible de confirmer, d'infirmer ou de compléter des hypothèses déjà étayées par d'autres travaux, mais aussi de refléter des conceptions pédagogiques, difficilement observables directement. Étudier des justifications par des statistiques lexicales permet en outre d'accéder à des représentations collectives. II ne s'agit donc pas d'affirmer que les normes langagières influent sur les normes d'action (ce qui est possible, mais que nous ne montrons pas ici), mais que les discours et les normes langagières qu'ils portent sont susceptibles de révéler l'hétérogénéité et la complexité des conceptions.

\section{L'enquête et la méthodologie d'analyse}

\subsection{Le questionnaire}

Le questionnaire ${ }^{2}$ proposait aux répondants dix-huit cas brefs, dont seize ont finalement été retenus pour l'analyse. À chaque fois, ils avaient à choisir dans un QCM la décision la plus proche de celle qu'ils prendraient eux-mêmes s'ils étaient confrontés à la situation de classe décrite (question fermée ${ }^{3}$ ). Ils devaient ensuite librement expliciter les raisons de leur choix (question ouverte ${ }^{4}$ ).

Les cas soumis aux répondants décrivent des situations réelles d'enseignementapprentissage dans différentes disciplines, choisies parce qu'elles mettent les enseignants en face de dilemmes, où des valeurs et des normes antagonistes sont à l'œuvre dans le processus de choix : par exemple, "décider d'avancer avec les forts ou de se concentrer sur les plus faibles" (Wanlin et Crahay, 2012, p. 9). Face à ces "choix quasi insolubles" mettant en concurrence des principes différents, les répondants sont amenés à justifier leurs choix et à les tempérer, en explicitant et en hiérarchisant ces principes, bref à expliciter ce qui sous-tend leurs décisions lorsqu'ils font la classe.

2 Ont contribué à la conception du questionnaire et/ou à sa passation, dans le cadre de Reseida (Réseau de recherche sur la socialisation, l'enseignement, les inégalités et les différenciations dans les apprentissages) : Annie Cabrera, Jacques Crinon, Catherine Delarue-Breton, Catherine Dupuy, Dominique Gelin, Georges Ferone, Éliane Fersing, Bruno Fondeville, Sabine Kahn, Corinne Marlot, Marie Toullec-Théry, Nathalie Sayac.

3 Les réponses au QCM ont été construites à partir de réponses réelles d'enseignants auxquels les cas avaient été soumis dans la phase préparatoire de l'enquête.

4 Voir un exemple de cas en annexe. 


\subsection{La population interrogée}

Les passations ont eu lieu entre 2012 et 2014, auprès d'enseignants de l'école primaire, lors de formations. Nous avons recueilli et soumis à l'analyse 163 questionnaires. Le tableau suivant indique la répartition des répondants selon différents critères.

\begin{tabular}{|l|l|c|}
\hline Maternelle & & 46 \\
\hline CP/CE1 & & 76 \\
\hline CE2/CM1/CM2 & & 67 \\
\hline En dispositif prioritaire & & 24 \\
\hline Maîtres formateurs & & 19 \\
\hline Ancienneté (en années) & Stagiaire en formation initiale & 18 \\
\cline { 2 - 3 } & 1 à 5 & 35 \\
\cline { 2 - 3 } & 6 à 10 & 28 \\
\cline { 2 - 3 } & 11 à 15 & 26 \\
\cline { 2 - 3 } & 16 à 20 & 26 \\
\cline { 2 - 3 } & Plus de 20 & 28 \\
\cline { 2 - 3 } & Non renseignée & 2 \\
\hline Femme & & 132 \\
\hline Homme & & 31 \\
\hline
\end{tabular}

Tableau 1 : Caractéristiques des répondants

\subsection{L’analyse lexicométrique}

Nous nous intéressons aux réponses libres justifiant les choix d'action envisagés face aux cas présentés. Ce corpus de discours est interrogé en ayant recours à des statistiques lexicales. Celles-ci procèdent par comptage (un corpus est alors caractérisé par la fréquence respective des mots qui le constituent), par comparaison entre des parties du corpus analysé, dont le lexique diffère, par l'étude du sens particulier des mots dans le corpus à travers leurs emplois (Lebart et Salem, 1994; Tournier, 1980).

Nous avons procédé à trois types d'investigations, en utilisant le logiciel Lexico (Lebart et Salem, 1994) :

- nous avons établi le lexique du corpus (les 163 "discours"), avec la fréquence de chaque mot dans l'ensemble de ce corpus. Cette liste permet une première exploration de la hiérarchie des préoccupations des répondants; 
- nous avons cherché à savoir si ce lexique comportait des spécificités en fonction des caractéristiques des répondants (répertoriées dans le tableau 1);

- nous avons tenté de mieux comprendre ce que les enseignants interrogés mettent sous les mots qu'ils emploient; aussi avons-nous choisi deux mots parmi les plus fréquents dans ce corpus (élèves et temps), établi leurs concordances (l'ensemble de leurs contextes d'apparition), puis classé ces contextes selon des critères syntaxiques, les relations syntaxiques permettant d'accéder à des relations sémantiques que nous nous sommes efforcés de mettre en évidence.

\section{La fréquence des mots}

Si l'on ne tient pas compte des termes dont la forte fréquence se retrouve d'une manière générale dans les corpus linguistiques, et qui ne disent rien en soi de l'univers mental des répondants (déterminants, pronoms personnels, double négation etc.), apparaissent le plus fréquemment les termes élèves (1 138 occurrences $^{5}$, terme attendu dans ce corpus), travail (308 occurrences), mais aussi peut (417 occurrences), faire (349 occurrences), être (352 occurrences), temps (255 occurrences), enseignant (213 occurrences). Ces termes sont certes pour partie induits par la formulation du questionnaire mais nous semblent traduire aussi des normes et valeurs emblématiques de la communauté enseignante contemporaine, centrées davantage sur l'action et les contraintes de l'action que sur le savoir, nous y reviendrons.

\section{Des sous-groupes aux discours distincts?}

L'étude des fréquences relatives des mots dans les sous-populations correspondant à une caractéristique particulière $^{6}$ (voir le tableau 1) indique que seul le groupe des formateurs se distingue. Exercer des fonctions de formateur a une incidence sur les termes privilégiés pour évoquer la pratique de la classe. Les formateurs n'emploient jamais difficile et tard, ce qui permet d'envisager qu'ils s'inscrivent dans un autre rapport au temps, peut-être moins monolithique, que leurs collègues non formateurs. Ils évoquent plus rarement que les non-formateurs le matériel. Ils utilisent significativement plus que les autres enseignants les mots suivants : note, paraît, stratégie, situation, propose, obstacles, permettre, phénomène dont on peut supposer qu'il reflète les différentes formes de diffusion ou de vulgarisation des savoirs scientifiques dans la sphère de la formation des enseignants. Les termes stratégie

5 Il faut en outre y ajouter la forme élève, qui apparait 152 fois.

6 C'est ce qu'on appelle spécificités par parties en statistique lexicale. 
ou obstacles sont en effet présents dans la recherche en éducation, et les termes propose ou permettre supposent un élève en action, à qui est dévolue une part de l'activité.

En revanche, les autres caractéristiques étudiées n'influent pas sur le vocabulaire employé, contrairement à ce que nous pouvions imaginer. L'ancienneté dans le métier ne conduit pas les répondants à utiliser les mots avec des fréquences différentes. II n'apparait pas non plus de différence entre les enseignants d'éducation prioritaire et ceux qui exercent hors éducation prioritaire. Ce constat nous conduit à penser que les conceptions générales que les enseignants ont de l'action pédagogique sont indépendantes du contexte d'exercice et de l'ancienneté dans la carrière.

\section{Ce que les mots veulent dire}

Nous avons choisi de restreindre ici notre étude aux emplois de deux mots parmi les plus fréquents dans le corpus : élève(s) et temps. Afin d'étudier ces emplois, nous avons systématiquement relevé les contextes de ces formes-pôles, classés selon la relation syntactico-sémantique entre la forme-pôle et ces éléments de contexte, puisque, comme nous l'avons indiqué dans la présentation de la méthodologie, les contextes d'emploi permettent d'expliciter le sens que les enquêtés donnent aux mots.

\section{1. Élèves/élève}

\subsubsection{Déterminants et expansions du nom}

On trouve le plus souvent "les élèves". Avec deux emplois principaux de l'article défini pluriel, qui traduisent la coexistence de deux visions de l'élève.

Dans un premier emploi, "les élèves" englobe l'ensemble des élèves de la classe, voire des élèves en général (ce qui rejoint alors le syntagme "l'élève" au singulier) : les élèves forment un groupe, un ensemble aux réactions et aux besoins communs ${ }^{7}$. C'est l'emploi le plus fréquent (481 occurrences). II subsiste cependant une ambigüité, car ce syntagme désigne soit le groupe auquel on s'adresse, par exemple pour fixer des consignes de travail, et qu'il s'agit de gérer (par exemple : "j'aurais mis les élèves par deux"), soit les élèves abstraits, dans une conception indifférenciée d'un public homogène (notamment lorsqu'est énoncée une sorte de maxime : "il faut être ambitieux pour les élèves"). Les élèves, ce sont alors les élèves auxquels l'enseignant adapte le rythme du travail et les exigences, le steering group décrit par la littérature (Wanlin et Crahay, 2012).

7 On pense ici aux observations de Bromme (1987). 
Dans un deuxième emploi, le syntagme "les élèves" est accompagné d'un adjectif déterminatif (ou d'une relative déterminative, ou d'un complément déterminatif) qui restreint l'extension du nom : par exemple, "les élèves perdus" (2 occurences), "les élèves en difficulté(s)" (44), "les bons élèves" (49), "les élèves qui n’ont pas travaillé" (2). Il désigne alors une catégorie d'élèves, opposée à d'autres, pour déterminer par exemple ce qui est possible avec certains et pas avec d'autres. Cet emploi, moins fréquent (et qui réfute d'ailleurs parfois la pertinence d'une distinction entre élèves : "si j’interroge les élèves ayant la bonne réponse, nous n'aurons pas d'hypothèses erronées et je ne connaitrai pas les représentations des autres élèves"), semble être propre au discours de certains enseignants, plus sensibles à l'hétérogénéité qu'à l'homogénéité de leur public. De manière massive, distinguer les élèves, c'est distinguer les "bons élèves" (49) et les "élèves en difficulté(s)" (44) ou encore "faibles" (3) ou "fragiles" (1), les distinctions fondées sur l'activité cognitive des élèves dans la classe restant beaucoup plus rares.

Fréquent aussi est le syntagme "tous les élèves" (et ses équivalents : "l'ensemble des élèves"). Nous y reviendrons plus loin lorsque nous examinerons les verbes et prédicats associés à ce syntagme : "mon objectif est que tous les élèves puissent suivre".

Certains emplois individualisent les élèves : le répondant s'intéresse à des élèves particuliers. Il s'agit le plus souvent de ramener la réflexion sur "les autres élèves" "les élèves qui..., les autres...").

La plupart des déterminants (ou segments équivalents à des déterminants) précédant "élève(s)" renvoient à la préoccupation du tout et de la partie, du nombre d'individus concernés par rapport au tout de la classe. Cette manière de penser le dilemme du tous et du chacun est clairement formulée par certains enseignants ("S'il n'y a qu'un élève qui pose la question, je lui donne le sens du mot. Si plusieurs élèves posent la même question, alors je renvoie la question à l'ensemble des élèves."), mais elle est donc présente massivement à travers ces emplois récurrents des déterminants.

\subsubsection{Actions des élèves}

Au-delà des déclarations abstraites, voire stéréotypées sur l'activité des élèves ("Les élèves doivent être acteurs de leur apprentissage"...), que font les élèves mis en scène par nos enseignants?

Une première remarque est que, dans le large éventail des actions prêtées aux élèves (lire, écrire, écouter, réaliser un exercice, travailler, choisir, chercher, verbaliser, passer au tableau...), les plus fréquentes sont celles de comprendre (27), d'interroger ou de s'interroger (9).

Une seconde remarque est que les propositions où les élèves sont sujets (sémantiquement) sont souvent insérées dans des structures à valeur normative indiquant ce qu'ils doivent faire : "les élèves doivent comprendre que"... Encore plus souvent, ces actions sont modalisées par le verbe "pouvoir": "les élèves peuvent mieux appréhender la notion 
difficile". Ces formules manifestent des modalités énonciatives de l'ordre de l'incertain, qui laissent apparaître un scepticisme sur sa propre efficacité d'enseignant à mettre les élèves au travail ou leur permettre de progresser, ou un doute devant l'énigme de ce qui se passe dans la tête des élèves et pourraient ainsi manifester une difficulté à prendre des décisions à partir de ce qu'ils perçoivent des élèves (Crahay et Wanlin, 2012).

\subsubsection{Actions sur les élèves}

Les cas où "élève(s)" est objet sont moins nombreux que ses emplois comme sujet. Presque toujours, les élèves sont alors l'objet de l'action de l'enseignant, qui les interroge (14) ou questionne (1), les aide (13), les guide (5), les fait passer au tableau (6), craint de les perdre (3) / larguer (1) / bloquer (1), les regroupe (1), enrôle (1), recentre (1), aiguille (1), implique (1), valorise (1), met en situation de réussite (2)...

Dans d'autres cas, les élèves sont les bénéficiaires de l'action (là encore de l'enseignant) : il leur montre (4), donne la parole (2), demande (3)...

Mais "élève(s)", très souvent aussi, est employé dans des constructions avec "faire" (12), " permettre" (11), "laisser" (6), "demander" (4), "inciter" (1), "inviter" (1), etc., au sein desquelles il apparait comme un sujet manipulé, soit directement par l'enseignant (qui " fait verbaliser les élèves", "fait interagir les élèves"...), soit par la situation que l'enseignant a créée ("cette solution permet aux élèves de confronter leurs hypothèses").

\subsubsection{Autres syntagmes nominaux complétés}

Les substantifs complétés sont souvent des termes qui concernent les représentations ou conceptions des élèves (12 occurrences), donc implicitement considérées comme des idées fausses qu'il faut remplacer par des idées plus justes - ce phénomène apparait clairement lorsque les enseignants parlent de s'appuyer sur les erreurs des élèves -, et moins souvent les connaissances ou savoirs (7 occurrences) sur lesquelles l'enseignant pourrait s'appuyer pour faire acquérir d'autres savoirs. Aussi les répondants insistent-ils souvent sur une démarche partant du négatif (par exemple : "Je pense qu'il faut partir des difficultés des élèves"), sur les déficits, plutôt que sur le positif. En tout cas, ce sont deux types de mise en scène des élèves qui opposent deux manières de voir dans notre population d'enseignants ("Il est préférable d'utiliser les connaissances des élèves" vs "Il faut partir des difficultés des élèves, parvenir à les leur faire exprimer"). 


\subsection{Temps}

\subsubsection{Déterminants et expansions du nom}

Si l'on excepte deux utilisations de "temps" pour désigner une époque historique ("notre temps"), "temps" renvoie au temps de la classe. Deux emplois peuvent être distingués. D'une part, ce terme rend compte de la succession des moments de classe : "les temps" (4), "un temps" (29), "ce temps" (8), et surtout "un premier temps" (20), opposé à "un second temps" (7) ou "un deuxième temps" (6). Le temps est alors le temps opportun. Il y a un ordre des moments scolaires, et ces temps successifs structurent le travail des élèves.

D'autre part, une autre acception du terme concerne le temps-durée : "du temps" (31); c'est le temps dont l'enseignant subit la pression, parce qu'il manque ou qu'il est difficile à doser justement : "plus de temps" (14), "peu de temps" (3), "trop de temps" (8). Face à des horaires toujours insuffisants pour ce qu'il a prévu, l'enseignant ressent le dilemme : ne pas passer "trop de temps" et donner "plus de temps". D’autant plus que plusieurs des répondants explicitent une conception des élèves comme des fruits qui murissent sans qu'on ait à faire plus que d'attendre : "Des fois, le temps suffit et est un facteur à la compréhension des notions".

Ces deux emplois viennent parfois se recouvrir : "un temps plus long est nécessaire", car le dosage de la durée des moments de travail successifs est aussi une question qui se pose.

\subsubsection{Actions du temps}

"Temps" en position de sujet est rare. Là encore, on peut distinguer le temps-moment (qui "permet" (5) ou non une tâche ou une avancée) et le temps-durée, qui "manque" (2), "suffit" ou non (3), "débloque" ou non une situation (2), et contraint l'enseignant "toujours pressé par le temps".

\subsubsection{Actions sur le temps}

Plus fréquents sont les emplois de cette forme en position d'objet. II s'agit alors du temps-durée : "avoir le temps" (8), "perdre du temps" (22), "gagner du temps" (6), etc.

Cette analyse confirme que deux grands types de rapports au temps, conflictuels mais inscrits cependant tous deux dans une conception linéaire, se distinguent : "prendre le temps de" et "ne pas perdre de temps". 


\subsubsection{Autres syntagmes nominaux complétés}

L’inventaire des substantifs complétés confirme la préoccupation à gérer le temps : "le facteur temps", "la contrainte temps", "contrainte de temps", "manque de temps", même s'il ne s'agit que d'une occurrence à chaque fois.

\section{Discussion}

\subsection{Un lexique de l'action et du dispositif plutôt que du savoir}

Le lexique utilisé (fréquence de faire ou travail), ainsi que les manières de caractériser les élèves, laisse apparaître une centration sur les modes de réalisation des tâches scolaires et de l'activité où le savoir, relégué à l'arrière-plan, n'est que très peu mis en avant pour mieux comprendre et prendre en charge les obstacles susceptibles d'entraver leur appropriation par les élèves, ce qui vient confirmer des travaux antérieurs (Bautier et Rayou, 2009; Delarue-Breton, 2012; Rochex et Crinon, 2011). Nous formulons en outre ici I'hypothèse du paradoxe d'une forme de vulgarisation des recherches issues des didactiques disciplinaires (qui placent pourtant au centre de leur ancrage scientifique la spécificité des savoirs convoqués), qui conduirait les enseignants à privilégier le dispositif lui-même, au détriment des propriétés conceptuelles dont il est supposé favoriser l'acquisition. Les élèves doivent ainsi agir eux-mêmes (élève agent), l'enseignant doit les faire agir (élève manipulé), ou encore agit pour eux (élève bénéficiaire de l'action), mais toujours dans le but de les rendre eux-mêmes acteurs; cette mise en action des élèves entraîne une série de difficultés et de contraintes inhérentes à l'action, susceptibles de détourner l'intérêt de l'enseignant des contraintes inhérentes aux savoirs enseignés, ou plus largement aux contenus.

\subsection{La contrainte temporelle}

En ce qui concerne la question du temps, et plus particulièrement dans son acception de durée, celui-ci semble davantage appréhendé à partir d'une conception chronologique (qu'il s'agisse du temps "durée" ou des temps "moments"), que dans sa complexité. Dans la note de synthèse qu'il a consacrée à la question des temporalités éducatives, Lesourd (2006) montre que cette notion implique pourtant un double rapport du temps à l'éducation : à la fois condition de l'action éducative et objet de praxis éducative, la temporalité éducative revêt un caractère simultanément agissant et agi, qui suppose d'envisager sa double dimension déterminante et déterminée; or, qu'il s'agisse d'en prendre, d'en gagner ou de ne pas en perdre, il n'est que très peu question, dans ce que nous observons dans 
notre corpus, de le construire, ce qui, ici encore, va à l'encontre des réflexions conduites par les didacticiens sur la conception des dispositifs, qui impliquent une orchestration des différentes temporalités (Chopin, 2010; Reuter, 2013).

On observe par ailleurs que le temps évoqué est peu envisagé du point de vue des élèves : contrainte forte de l'action pédagogique (ne pas perdre de temps), d'ailleurs souvent déjà relevée dans le discours des enseignants (Chopin, 2010), le temps est parfois envisagé pour les élèves (leur donner plus de temps, leur faire gagner du temps), mais jamais dans la manière dont il est susceptible d'être éprouvé par ceux-ci : temps enfermants, extérieurs et coercitifs soumis à la nécessité de l'urgence chronique (Grossin, 1996), temps qui s'étire indéfiniment (Bernstein, 2007).

La contrainte temps apparait donc envisagée comme une contrainte externe, diachronique (temps successifs), sur laquelle l'enseignant n'a que peu de prise, et peu dans sa dimension synchronique (temps coprésents).

\subsection{Lexique d'une école pérenne et lexique contemporain}

D’une manière générale, on observe l'emploi de termes dont on peut considérer qu'ils relèvent tantôt d'un vocabulaire scolaire contemporain (comme procédures, stratégies, compréhension fine), tantôt d'un vocabulaire plus classique ou plus traditionnel (comme réponse, travail, exercice). Cette concomitance d'usages invite à considérer que le discours des enseignants est constitué de termes qui renvoient à ce que l'on pourrait appeler des valeurs sures, stables, susceptibles de traverser les époques et les contextes, et de termes qui disent quelque chose de ce que l'on pourrait nommer les "normes ambiantes".

En particulier, parmi les termes relevant d'un vocabulaire contemporain, on note l'emploi fréquent du mot compréhension (218 occurrences) et, parmi les verbes associés à élèves, comprendre, le domaine de la compréhension constituant un objet de recherche qui apparait au carrefour de divers champs disciplinaires scientifiques et qui fait partie des domaines susceptibles de contribuer largement aux difficultés récurrentes des élèves, les difficultés liées au champ de la compréhension ayant un impact sur l'ensemble des apprentissages. Cela pourrait contribuer à rendre compte de la primauté du lexique du comprendre sur celui de l'apprendre.

\subsection{Des stéréotypes langagiers (un "politiquement correct") pour parler des élèves}

En quoi les normes langagières (ce qu'il est possible, nécessaire, permis ou interdit de dire, ou ce que l'on croit possible, nécessaire, permis ou interdit de dire) révèlent-elles 
des manières d'envisager la pratique professionnelle, indépendamment de la pratique elle-même?

L'étude des contextes des termes employés pour désigner et caractériser les élèves nous invite à penser que les changements de dénomination peuvent n'être que des changements de surface, susceptibles de révéler une forme enseignante (et institutionnelle) de politiquement correct, qui viendrait cependant masquer des conceptions de l'élève et du métier peut-être moins évolutives qu'il n'y parait. Nous formulons ici l'hypothèse d'une tension vive entre prescription institutionnelle imprégnée de discours didactiques, et plus généralement scientifiques qui circulent dans la sphère scolaire, et difficulté des enseignants à prendre eux-mêmes en charge les difficultés croissantes du métier auxquelles ils sont confrontés. L'étude des déterminants du mot élève notamment, qui montre une forme d'indifférenciation dans la désignation des élèves, y compris dans la dichotomie entre bons et fragiles, suggère en filigrane une conception de l'élève caractérisée par une hétérogénéité peu saisissable, peu classable, et par conséquent peu maitrisable.

Cette réflexion invite à considérer l'emploi de certains termes comme traduisant des normes verbales susceptibles de contribuer à la définition du groupe social que constitue le corps enseignant, et à tenter de mieux comprendre certains changements de dénomination qui disent selon nous quelque chose des interdits implicites qui traversent potentiellement ces discours, plutôt que des changements de conception à part entière.

Notamment, si la formule "bons élèves" est fréquente - avec ou sans guillemets, ce qui mérite aussi de s'y arrêter - on ne rencontre pas le pendant "mauvais élève(s)", même avec guillemets. Des formules de substitution, du type "élèves en difficulté", "élèves fragiles", "les plus fragiles" etc. sont cependant récurrentes dans le corpus.

La présence récurrente des guillemets dans l'appellation "bons élèves", ou dans le syntagme "les bons" nous invite à penser que ce type même de catégorisation n'est pas totalement légitime, même quand elle n'est pas négative. On peut y voir une forme de déplacement des conceptions valorisées, notamment par l'institution, qui visent à ne plus enfermer les élèves, peut-être grâce à l'influence de nombreux travaux scientifiques montrant les dangers de ce type d'essentialisation (en psychologie sociale notamment), dans des catégories dont ils ne s'échappent plus. La conception de l'élève acteur comme principe en soi ou valeur partagée fonctionnerait ainsi davantage comme un emblème, un étendard de l'appartenance au groupe social des enseignants, mais peu comme principe d'action.

Par ailleurs, nous notons que les formateurs n'emploient à aucun moment les termes "tard" et "difficile"; nous avons suggéré précédemment que cette spécificité pouvait indiquer l'existence d'une autre conception du temps (moins monolithique) ou de l'élève (qui se développe dans le temps); nous suggérons cependant ici une autre éventualité (qui n'est pas incompatible avec celle d'une conception moins monolithique du temps), celle d'une plus grande appropriation des stéréotypes langagiers par les formateurs, qui connaissent mieux que les enseignants ordinaires les prescriptions institutionnelles de tous ordres. 
Ces différents stéréotypes langagiers nous amènent à considérer que, pour une part non négligeable des enseignants, la manière dont l'enseignant se constitue comme ressource pour les élèves ne s'inscrit pas dans un projet pédagogique dont la visée première serait leur émancipation. Ainsi, si l'action prônée par certains d'entre eux doit conduire les élèves à s'approprier des ressources de natures diverses pour apprendre, il semble que d'autres se constituent eux-mêmes comme ressources pour les élèves. Ce constat invite à mettre en avant le caractère paradoxal d'un discours ambivalent qui, d'un côté, met en avant une conception de l'élève acteur, qui construit lui-même ses apprentissages, éventuellement tout seul, mais qui par ailleurs suggère une conception moins assumée de la relation pédagogique, au sein de laquelle les élèves - et en particulier "les plus fragiles d'entre eux" - s’inscrivent dans la dépendance nécessaire envers l'enseignant.

\section{Conclusion}

Dans un article consacré aux mutations de la normativité dans l'école contemporaine, et plus particulièrement dans l'enseignement secondaire, Verhoeven (2012) évoque, à propos de la manière de gérer l'ordre scolaire, un "mouvement de déstabilisation normative" (p. 98). Cette hypothèse d'une déstabilisation normative vaudrait également, nous semble-t-il, pour le premier degré et pour rendre compte de la manière de concevoir la transmission des savoirs, au cœur des cas sur lesquels les enseignants de notre enquête ont été invités à réagir.

En effet, notre analyse nous conduit à conclure à un émoussement des certitudes communes. Si des formules, des mots d'ordre, des références communes sont affirmés (l'élève acteur en particulier), celles-ci recouvrent cependant des manières contrastées de dire et de penser l'acte d'enseignement. D’autres références différencient immédiatement les enseignants interrogés (sur la prise en compte du temps didactique en particulier). Certes, ces contradictions renvoient à des dilemmes constitutifs du métier : dans un enseignement collectif, sur quels élèves régler le rythme du travail? Comment susciter et maintenir l'adhésion et l'engagement de tous les élèves dans les tâches proposées? Mais ce qui apparait ici est bien un éclatement des repères et une juxtaposition de références disparates. Nos analyses invitent ainsi à envisager ce feuilletage normatif comme reflet possible d'une certaine inquiétude vis-à-vis du métier, quand ce qui est demandé aux enseignants pour tenter de résorber les inégalités scolaires est d'une complexité croissante.

Ce feuilletage interne n'est cependant pas - et il est nécessaire de le souligner - la seule forme de l'hétérogénéité normative qui traverse l'activité enseignante : celle-ci coexiste avec une hétérogénéité entre enseignants, et le recours parfois à certaines formules emblématiques ou "étendards", qui pourraient apparaître comme des mots d'ordre, des points de rassemblement de la profession, sont sans doute à considérer également comme des tentatives de tenir ensemble des points de vue très éclatés. Autrement dit, il est possible 
de penser que l'absence de normes réellement partagées par l'ensemble de la profession, qui se voit en outre confrontée à la nécessité de faire face à un métier de plus en plus complexe, suscite ce besoin de mots d'ordre ou formules emblématiques.

En termes de formation, c'est dès lors l'analyse conjointe, avec les enseignants, de la nature et de la complexité des normes implicites qui sous-tendent leur action, comme nous avons tenté de le faire ici, qui nous semble utile pour construire un rapport plus lucide aux objectifs poursuivis et aux moyens de les atteindre, plutôt que la diffusion des résultats de la recherche.

Jacques Crinon

jacques.crinon@u-pec.fr

Catherine Delarue-Breton

catherine.delarue-breton@univ-rouen.fr

\section{Bibliographie}

Bautier, É. et Rayou, P. (2009). Les inégalités d'apprentissage. Programmes, pratiques et malentendus scolaires. Paris : PUF.

Bernstein, B. (2007). Pédagogie, contrôle symbolique et identité. Théorie, recherche, critique. Laval, Canada : Presses de l'université Laval.

Bromme, R. (1987). Teachers' assessments of students' difficulties and progress in understanding in the classroom. Dans J. Calderhead (dir.), Exploring teachers' thinking (p. 125-146). Londres, Royaume-Uni : Cassel.

Buisson-Fenet, H. et Dutercq, Y. (2015). Les cadres de l'encadrement : la gouvernance intermédiaire des systèmes éducatifs en question. Recherche et formation, 78, 9-18.

Chopin, M.-P. (2010). Les usages du "temps" dans les recherches sur l'enseignement. Revue française de pédagogie, 170, 87-110.

Clot, Y. (2010). Le travail à coeur. Paris : La Découverte.

Crahay, M., Wanlin, P., Issaieva, I. et Laduron, I. (2010). Fonction, structuration et évolution des croyances (et connaissances) des enseignants. Revue française de pédagogie, 172, 85-129.

Delarue-Breton, C. (2012). Discours scolaire et paradoxe. Louvain-la-Neuve, Belgique; Paris : Academia; L'Harmattan.

Delarue-Breton, C. (2016). Écriture scientifique et évolution des croyances, des connaissances ou des conceptions des enseignants en reprise d'étude. SHS Web of Conferences, 27. Récupéré sur le site de la revue : <https://www.shs-conferences.org/articles/shsconf/pdf/2016/05/shsconf_ cmlf2016_06005.pdf>.

Delarue-Breton, C. et Dolignier, C. (2016). Posture "seconde" et évolution des croyances des enseignants en formation initiale : le rôle de la recherche. Dans B. Marin et D. Berger (dir.), Recherches en éducation, recherches sur la professionnalisation : consensus et dissensus. Le printemps de la recherche en ESPE 2015 (p. 194-204). Paris : Réseau national des ESPE.

Détrie, C., Siblot, P. et Verine, B. (2001). Termes et concepts pour l'analyse du discours : une approche praxématique. Paris : Honoré Champion. 
Goigoux, R. (2007). Un modèle d'analyse de l'activité des enseignants. Éducation et didactique, 1(3), 47-69. Grossin, W. (1996). La notion de milieu temporel. Temporalistes, 32, 20-26.

Lebart, L. et Salem, A. (1994). Statistique textuelle. Paris : Dunod.

Lesourd, F. (2006). Des temporalités éducatives : note de synthèse. Pratiques de formation, 51-52, 9-72. Nonnon, É. et Goigoux, R. (2007). Travail de l'enseignant, travail de l'élève dans l'apprentissage initial de la lecture. Repères, 36, 5-36.

Reuter, Y. (éd.). (2013). Dictionnaire des concepts fondamentaux des didactiques (3e éd.). Louvain-laNeuve, Belgique : De Boeck.

Rochex, J.-Y. et Crinon, J. (2011). La construction des inégalités scolaires. Au cœur des pratiques et des dispositifs d'enseignement. Rennes: PUR.

Tournier, M. (1980). D’où viennent les fréquences de vocabulaire? La lexicométrie et ses modèles. Mots, 1, 189-209.

Wanlin, P. et Crahay, M. (2012). La pensée des enseignants pendant l'interaction en classe. Une revue de la littérature anglophone. Éducation et didactique, 6(1), 9-46.

Verhoeven, M. (2012). Normes scolaires et production de différences. Les sciences de l'éducation. Pour l'ère nouvelle, 45(1-2), 95-121.

\section{Annexe}

\section{Un exemple de cas tiré du questionnaire}

\section{CAS 7}

Au cours d'une leçon de grammaire sur les propositions subordonnées relatives, quelques élèves sont perdus. L'enseignant se rend compte qu'ils ne mettent aucune signification sous les termes de sujet, de verbe et de phrase simple, alors que les notions sont supposées acquises depuis longtemps.

1. L'enseignant improvise pour toute la classe un travail de révision sur ces catégories grammaticales.

2. L'enseignant divise la classe en deux groupes. L'un des groupes étudie comme prévu la proposition relative, l'autre étudie de nouveau la structure sujet verbe de la phrase simple.

3. L'enseignant continue avec toute la classe un travail sur la proposition relative et diffère un travail spécifique avec les élèves en difficulté.

Quelle est, parmi ces manières de faire de l'enseignant, celle qui est la plus proche de la décision que vous prendriez?

Justification de votre choix : 


\section{Abstract \\ Norms and values in the discourse of primary school teachers: plurality and internal contradictions}

ABSTRACT: The norms which underlie what teachers do and think result from a compromise between external norms and their beliefs. We study these norms by means of a questionnaire-based survey of 163 primary school teachers. We analyse the responses to open questions through the words used by the teachers, using lexical statistics. The use of the words "élèves" (students) and "temps" (time), which occur very frequently in the responses, reveal several contradictions, which are explained both by the dilemmas inherent in the job and an explosion of benchmarks.

KEYWORDS: teacher, learning standard, speech, primary school, teaching practice 